\title{
Brain Oscillations Mediate Successful Suppression of Unwanted Memories
}

\author{
Gerd T. Waldhauser ${ }^{1}$, Karl Heinz T. Bäuml ${ }^{2}$ and Simon Hanslmayr ${ }^{1,3}$ \\ ${ }^{1}$ Department of Psychology, University of Konstanz, Konstanz, Germany, ${ }^{2}$ Department of Experimental Psychology, \\ Regensburg University, Regensburg, Germany and ${ }^{3}$ School of Psychology, University of Birmingham, Birmingham, UK
}

Address correspondence to Gerd Waldhauser, Department of Psychology, University of Konstanz, Postfach 905 , 78457 Konstanz, Germany. Email: gerd.waldhauser@uni konstanz.de

To avoid thinking of unwanted memories can be a successful strategy to forget. Studying brain oscillations as measures of local and inter-regional processing, we shed light on the neural dynamics underlying memory suppression. Employing the think/no-think paradigm, 24 healthy human subjects repeatedly retrieved (think condition) or avoided thinking of (no-think condition) a previously learned target memory upon being presented with a reminder stimulus. Think and no-think instructions were delivered by means of a precue that preceded the reminder by $1 \mathrm{~s}$. This allowed us to segregate neural control mechanisms that were triggered by the precue from the effect of suppression on target memory networks after presentation of the reminder. Control effects were reflected in increased power in the theta $(5-9 \mathrm{~Hz})$ frequency band in the medial and dorsolateral prefrontal cortex and higher long-range alpha (10-14 Hz) phase synchronization. Successful suppression of target memories was reflected in a decrease of theta oscillatory power in the medial temporal lobes and reduced long-range theta phase synchronization emerged after presentation of the reminder. Our results suggest that intentional memory suppression correlates with increased neural communication in cognitive control networks that act in down-regulating local and interregional processing related to memory retrieval.

Keywords: brain oscillations, forgetting, inhibitory control, memory suppression, think/no-think

\section{Introduction}

To intentionally avoid and suppress particular mental repre sentations is a powerful capability of the human mind. There is accumulating evidence that this ability extends to the episodic memory domain (Anderson and Huddleston 2012). The inten tional suppression of unwanted memories may be a successful strategy to forget what we prefer not to think about (Anderson and Green 2001; but see, e.g., Bulevich et al. 2006).

In the laboratory, the neurocognitive processes mediating forgetting by voluntary suppression can be investigated by means of the so called think/no think (T/NT) paradigm (Anderson and Green 2001). In the T/NT paradigm, partici pants are repeatedly cued to either suppress (NT condition) or retrieve ( $\mathrm{T}$ condition) memories when being presented with a reminder stimulus. On a behavioral level, such memory sup pression leads to forgetting of NT items when compared with never cued baseline items (Anderson and Green 2001; Hanslmayr, Leipold, et al. 2010; Waldhauser, Lindgren, et al. 2012). fMRI studies suggest that voluntary memory sup pression is the result of a dynamic interaction between two systems: (1) A prefrontal control system that down regulates (2) a distributed hippocampal/posterior cortical system, which presumably stores memory traces and their sensory representa tions (Anderson and Hanslmayr 2014; Anderson et al. 2004;
Depue et al. 2007; Benoit and Anderson 2012). Owing to their crucial role in orchestrating neural activity in distributed cell assemblies (Fries 2005), brain oscillations are strongly implicated during this interaction. To date, however, little is known about how brain oscillations mediate voluntary memory suppression.

In the present study, we re analyzed EEG data from a previ ously published event related potential study (Hanslmayr et al. 2009), where we utilized a modified T/NT paradigm. Therein, participants first studied face word pairs until a high learning criterion. In a subsequent $\mathrm{T} / \mathrm{NT}$ Phase, participants received an anticipatory T/NT Cue, which preceded the reminder (face) stimulus by $1 \mathrm{~s}$ (Fig. 1A). This spaced delivery of the T/NT Cue and the Reminder allowed us to separate the 2 aspects of memory suppression: The initiation of executive control in response to the T/NT Cue and the actual down regulation of storage related brain systems in response to the reminder. Employing time frequency analysis in terms of oscillatory power and phase synchronization as measured by EEG, we shed light on the dynamic oscillatory interaction of brain net works underlying voluntary memory suppression.

Like other forms of inhibitory control, memory suppression presupposes the recruitment of control mechanisms that act in the suppression of target networks (e.g., Aron 2007; Mecklin ger et al. 2009). In the EEG, these control mechanisms are evident in increased frontal theta oscillatory activity, which has been localized to medial and lateral prefrontal cortex regions during response conflict (Hanslmayr et al. 2008), memory interference (Staudigl et al. 2010), and memory suppression (Depue et al. 2013). Crucially, top down cognitive control in general and memory suppression in particular also rely on en hanced fronto parietal communication (Corbetta and Shulman 2002; Paz Alonso et al. 2013) that is reflected in increased phase synchronization in the alpha frequency band (Sauseng et al. 2005; Sadaghiani et al. 2012). Taken together, these findings suggest that memory suppression should in part be mediated by increased prefrontal theta $(59 \mathrm{~Hz})$ oscillatory power and higher phase coupling in the alpha (10 $14 \mathrm{~Hz})$ band.

Successful memory retrieval is usually reflected in increased oscillatory power in the theta band (Düzel et al. 2003; Osipova et al. 2006; Nyhus and Curran 2010). Previous studies demon strated that memory related theta power effects in the medial temporal lobe are volume conducted to the scalp and can be picked up by surface sensors, particularly at posterior sites (Guderian et al. 2009; Staudigl and Hanslmayr 2013). Retrieval and memory maintenance is also characterized by increased phase synchronization in the theta band, which appears to be generated by hippocampo cortical feedback loops (Guderian and Düzel 2005; Osipova et al. 2006; Cashdollar et al. 2009). Based on data showing that memory suppression decreases retrieval related hippocampal activity (e.g., Anderson et al. 

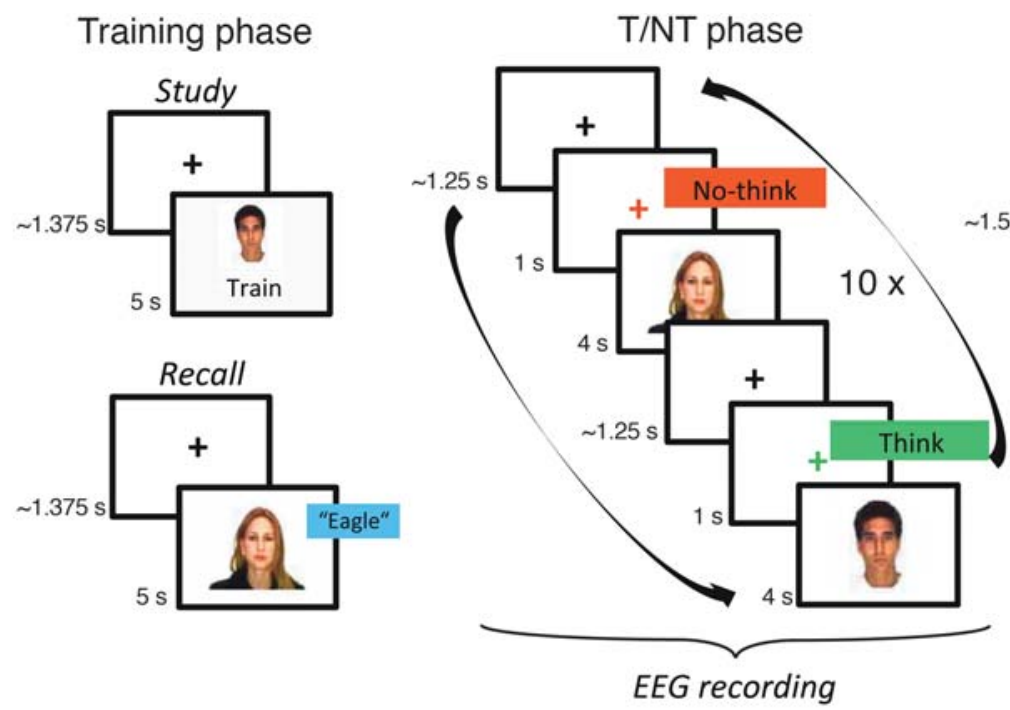

Recall

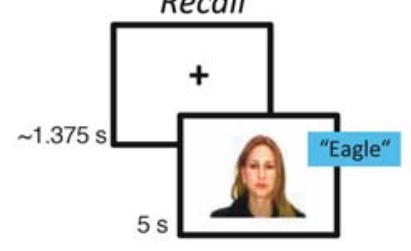

B

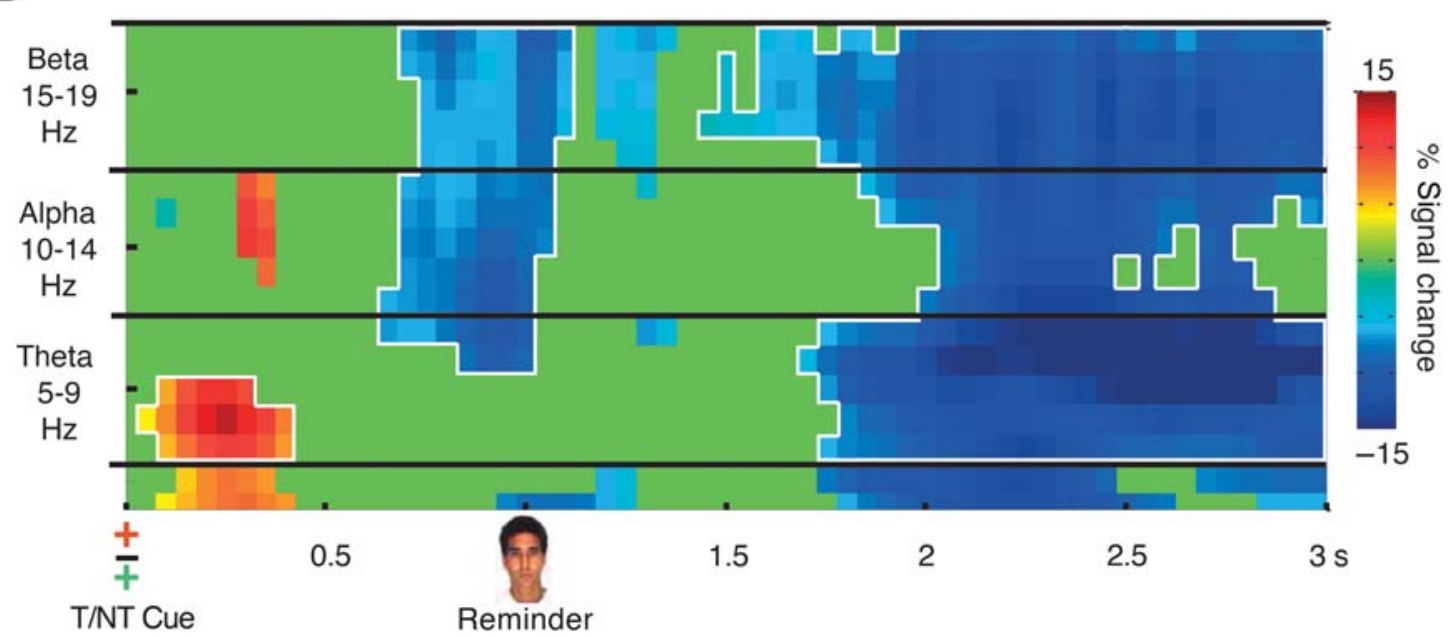

Figure 1. (A) Experimental procedure, trial sequence, and stimulus timing for Tand NT items, baseline items not shown. (B) Power difference between NT and T conditions (NT T), averaged across all electrode sites. Power values are masked with the results of a continuous Wilcoxon signed rank test over $10 \mathrm{~ms}$ and $1 \mathrm{~Hz}$ time frequency bins. Only differences with $P<0.05$ (one tailed) are shown. Significant clusters surviving permutation based correction are framed in white. Black lines indicate upper and lower borders of the frequency bands of interest.

2004), we expected the NT condition to be characterized by reduced theta power in posterior and medio temporal lobe regions and attenuated theta long range phase synchroniza tion. However, in addition to the expected decrease of theta oscillatory activity, memory suppression might also reflect de creased phase synchronization in the alpha frequency band. This would correspond to findings from encoding tasks, showing that decreased alpha phase synchronization correlates with lower memory performance (Bäuml et al. 2008; Hansl mayr et al. 2012). Thus, we expected an additional decrease of alpha synchronization as a marker of reduced retrieval, which is to be separated from increased synchronization in the same frequency range reflecting fronto parietal inhibitory control.

Two recent studies explored brain oscillatory correlates of voluntary memory suppression in the T/NT paradigm (Depue et al. 2013; Ketz et al. 2014). The study by Depue et al. (2013) reports an increase of theta and alpha oscillatory power in the NT condition when compared with the $T$ condition. In con trast, Ketz et al. (2014) attributed that theta power increases solely to the $\mathrm{T}$ condition, whereas memory suppression in the NT condition was characterized by a power increase in the alpha frequency range. These partly contradictory results could be due to the fact that both studies were unable to clearly separ ate the oscillatory correlates of increased prefrontal cognitive control from those related to the down regulation of the hippocampo cortical memory storage system. Also, phase syn chronization was not explored in both studies. Our modified T/ NT procedure allowed us to close these gaps by temporally seg regating cognitive control from memory suppression effects.

\section{Materials and Methods}

\section{Participants}

Twenty four volunteers (mean age: 21.5, range: 1925 years; 14 females) participated in the experiment. All participants were right handed, native German speakers, and had normal or corrected to normal vision. No participant reported any history of neurological disease. All subjects gave written informed consent and received course credits or monetary reward for participation.

\section{Materials}

Fifty four faces with a neutral expression were used as reminder stimuli, and 54 semantically unrelated words served as targets. 
The faces were drawn from the AR Face Database by Martinez and Be navente (1998). The word material was chosen such that each of the 54 words belonged to a different semantic category (Battig and Montague 1969; Mannhaupt 1983).

\section{Procedure}

The experimental session consisted of 2 blocks, each including a com plete T/NT procedure (training phase, T/NT phase, and testing phase; Depue et al. 2006; Hanslmayr et al. 2009). Accuracy was recorded by key press of the experimenter in all phases of the experiment.

\section{Training Phase}

In each of the 2 blocks, participants first went through 2 study test cycles (Fig. 1A, left). In each of the 2 cycles, participants studied 27 face word pairs including 3 filler items. This was followed by a cued recall test during which the face of a face word pair was presented as a reminder stimulus for the target word. Each study trial started with a fixation cross with variable duration $(1.251 .5 \mathrm{~ms})$, followed by a face word pair; participants were instructed to memorize the face word pairs. Each test trial consisted of a fixation cross with variable duration (1.25 $1.5 \mathrm{~ms}$ ), followed by a face; participants were instructed to overtly recall the associated target word when being presented with the face. Mean recall performance at the end of the second study test cycle was $80.75 \%(\mathrm{SD}=11.25)$ averaged across blocks; no participant recalled $<50 \%$ of the target items.

\section{Think/No Think Phase}

In each block, 8 faces of the 27 face word pairs were repeated 10 times during the T/NT phase and served as NT items, and another 8 face word pairs were repeated 10 times and served as $\mathrm{T}$ items. Eight face word pairs did not occur during the T/NT phase and served as a behavioral baseline. The material was counterbalanced across the 3 item types and the 2 blocks. To familiarize participants with the NT in struction before the T/NT phase, participants performed 15 NT prac tice trials during which each of the 3 filler items was repeated 5 times. For the NT trials, participants received a direct suppression instruction, to avoid thinking about the previously learned item by pushing the target item out of consciousness. The subjects were told to keep fix ation on the reminder stimulus, and they were discouraged from gener ating other associations to the reminder. Each trial in the T/NT phase started with a fixation cross, which turned either red or green after a variable delay ( $11.5 \mathrm{~s}$ ) to precue participants on whether to prepare for thinking of a previously studied item ( $\mathrm{T}$ : green) or prepare for not letting a previously studied item enter consciousness (NT: red; Fig. $1 A$, middle). The T/NT cue was followed by a face, which served as the re minder stimulus. The sequence of items was pseudorandomized, with the restriction that the same condition ( $T$ vs. NT) did not occur $>3$ times in a row.

\section{Test Phase}

During the test phase, all previously studied targets from the T, NT, and baseline conditions were tested by means of a cued recall task (Fig. $1 A$, right). A trial in the testing phase consisted of a fixation cross with variable duration (1.25 $1.750 \mathrm{~s}$ ), followed by the face of a previ ously studied cue target pair presented as a memory cue. Participants were instructed to recall and verbally report the word that was initially associated with the face during presentation of the memory cue.

\section{EEG Recording}

The EEG was recorded with a Brainamp MR+ amplifier (Megis, Brain Vision) from $63 \mathrm{Ag} \mathrm{AgCl} \mathrm{electrodes} \mathrm{positioned} \mathrm{according} \mathrm{to} \mathrm{the} \mathrm{ex}$ tended 1010 system. EEG was referenced to FCz during recording and digitally re referenced against the average of all electrodes. Vertical and horizontal eye movements were recorded by 2 additional channels to control for eye movements and blinks. Impedances were kept $<10$ $\mathrm{k} \Omega$, and the sampling rate was set to $500 \mathrm{~Hz}$. Signals between 0.1 and $250 \mathrm{~Hz}$ were recorded, and a notch filter was set at $50 \mathrm{~Hz}$. Before analysis, the EEG data were corrected for electro oculogram (EOG) artifacts using calibration data to generate individual artifact coeffi cients and the principal component analysis (PCA) method (for details, see Ille et al. 2002). All epochs containing remaining artifacts as identi fied by careful visual inspection were excluded from further analyses.

\section{EEG Analysis}

In contrast to our previous study, that is ERP study, on the same data set (Hanslmayr et al. 2009), we focused our analysis entirely on the contrast between T and NT conditions and high versus low forgetters collapsed across both halves of the TNT phase. This was done to be able to directly link our analyses to fMRI studies that typically employ a similar approach (e.g., Anderson et al. 2004). However, we cross checked our analyses with our previous approach (Hanslmayr et al. 2009) that focused on the second half of the TNT phase as the part of the experiment that most strongly results in successful memory sup pression. Second half data (not reported) yielded largely similar results as the present analysis using data collapsed across both halves.

Continuous EEG was epoched from 0.5 to $3 \mathrm{~s}$ around onset of the precue. EEG analysis was carried out in MATLAB (version R2010a; The MathWorks, Inc., Natick, MA, USA) using the FieldTrip toolbox (Oostenveld et al. 2011) and self written codes. We applied 21 Morlet wavelets, each with a width of 5 cycles of its center frequency period to derive a time frequency representation from the EEG signal from 1 to $20 \mathrm{~Hz}$. To avoid filter artifacts at the edges of the epochs, the data were filtered in a larger time interval, but analyses were restricted to the $3.5 \mathrm{~s}$ epochs. The resulting time frequency data were down sampled to $10 \mathrm{~ms}$ time bins to facilitate data handling.

\section{Sensor Level Analysis}

To quantify event related power modulations, poststimulus power change was calculated in relation to a baseline period ( 0.5 to $0 \mathrm{~s}$ precue; Pfurtscheller and Aranibar 1977). Phase synchrony between electrode sites was quantified as phase locking values (PLVs; Lachaux et al. 1999), using the phase estimates from the wavelet transformation. In order to minimize effects of volume conduction, current source density transformation was applied to the EEG data before PLV calcula tion using the CSD toolbox (http://psychophysiology.cpmc.columbia. edu/Software/CSDtoolbox/index.html; Kayser and Tenke 2006). PLVs were calculated for all possible pairs of electrodes in a frequency range from 1 to $20 \mathrm{~Hz}$. PLVs range from 0 to 1 , indicating maximal phase variability and maximal phase synchrony, respectively. The mea sure is independent of amplitude. The same amount of trials as for the condition containing the lower number of trials was randomly selected for the condition containing the higher number of trials, and phase es timates were averaged across 50 randomizations. This was done before calculating PLVs since trial numbers can bias phase synchronization measures.

\section{Source Localization}

We identified source activity for the obtained EEG power effects in the theta frequency band in order to gain a deeper understanding of their functional significance. We compared source activity between think and no think conditions (NT T) after correcting for baseline source ac tivity for each condition. In order to warrant stable estimation of sources and still allow for baseline correction with time frequency data uncontaminated by filter artifacts, time windows of $1 \mathrm{~s}$ length were chosen. Thus, power at the center frequency of $7 \mathrm{~Hz}$ was loca lized in time intervals of 01 and $1.52 .5 \mathrm{~s}$. The 2 time windows were chosen so that they should capture neural activity related to the differ ences of theta power between conditions in the interval following the TNT cue and the reminder. We expected generators of the early theta power increase in the NT condition to be localized in the medial and dorsolateral prefrontal cortex (PFC), corresponding to activity identi fied in fMRI studies on memory suppression (Anderson et al. 2004; Benoit and Anderson 2012). For the decreases of theta power, we ex pected generators in the medial temporal lobe (MTL) (Anderson et al. 2004; Benoit and Anderson 2012). It should be noted, however, that source localization of deep brain generators such as the hippocampus or MTL can be problematic (Quraan et al. 2011). Thus, we were pre pared to rather find reductions in cortical areas involved in memory re trieval and sensory processing such as the inferior parietal and visual cortices, potentially communicating with the hippocampal formation (Depue et al. 2007; Cabeza 2008). In addition, we also explored 
cortical sources of power differences between NT and T conditions in the alpha and beta frequency band. Our hypotheses concerning these frequency bands were less clear cut than for the theta band. As indi cated by studies on motor inhibition (e.g., Swann et al. 2009), we ex pected an interplay of alpha/beta power decreases in frontal areas as a marker of higher prefrontal cortical activity (Hanslmayr et al. 2011; Scheeringa et al. 2011) and increases in downstream areas such as the MTL reflecting inhibition of retrieval related processing areas (Wald hauser, Johansson, et al. 2012).

We employed the Dynamic Imaging of Coherent Sources (DICS) beamforming approach to identify sources of oscillatory activity after cal culating the cross spectral density matrix (Gross et al. 2001). Because trial numbers can bias source localization, values of the condition con taining the higher number of trials were averaged across 50 randomiza tions, selecting the same amount of trials as for the condition containing the lower number of trials. DICS constructs adaptive spatial filters to lo calize power for each grid point in the entire brain (Gross et al. 2001). For each individual and each time period of interest, filters were calcu lated using the baseline periods ( 1 to $0 \mathrm{~s}$ ) and activity of $1 \mathrm{~s}$ length fol lowing the precue or the reminder, including the trials from both conditions. The resulting average source estimate for each condition in the time intervals of interest was corrected for source activity in the base line interval and subsequently statistically compared with the other con dition. Statistical results of the comparison between conditions were interpolated to the standard Montreal Neurological Institute (MNI) brain.

\section{Statistical Methods}

The analysis of the behavioral and EEG data was based only on those face word pairs for which participants could recall the target item cor rectly in the second study test cycle of the training phase (Anderson et al. 2004; Waldhauser, Lindgren, et al. 2012). For statistical compari sons of all behavioral data, one tailed $t$ tests with the $\alpha$ level set to 0.05 were used.

Our EEG analysis focused on 3 frequency bands spanning a range of $5 \mathrm{~Hz}$ around a center frequency: theta $(59 \mathrm{~Hz})$ centered around 7 $\mathrm{Hz}$, alpha $\left(\begin{array}{ll}10 & 14 \mathrm{~Hz}\end{array}\right)$ with a center of $12 \mathrm{~Hz}$, and lower beta (15 19 $\mathrm{Hz}$ ) around $17 \mathrm{~Hz}$. This selection was based on statistically guided in spection of EEG power. This initial screening was done to identify po tential time windows of interest. In order to prevent that selection of time windows is biased by false positive results, we conducted a cluster based permutation test. In a first step, we identified clusters of adjacent time frequency bins with a significant $(P<0.05)$ difference between $\mathrm{T}$ and NT conditions by means of running Wilcoxon signed rank tests, averaged across all electrode sites (Fig. 1B). Second ly, we randomly swapped the values for T and NT conditions across subjects in 1000 randomizations. In analogy to the original data, clus ters of adjacent significant time frequency bins were identified. Thirdly, the cluster sizes (i.e., number of adjacent significant time fre quency bins) of the original data were tested against the cluster size distribution from the 1000 randomizations, such that only clusters bigger than random clusters at a predefined alpha level were consid ered significant (see Maris and Oostenveld 2007, for a similar ap proach). Alpha levels were adapted with a step wise Bonferroni correction, dividing an alpha level of 0.05 by the number of compari sons. Thus, the first cluster of the real data was considered significant when it was bigger than $95 \%$ (uncorrected) of the randomized clusters, the second cluster was considered significant when it was bigger than $97.5 \%$ of the second largest clusters $(P<0.05 / 2)$, given that a first cluster was identified in the randomization procedure, and so forth. Based on these results, both power and PLV were analyzed in 3 time windows with a minimum length of $0.5 \mathrm{~s}(00.5,0.51$, and $1.53 \mathrm{~s}$ after onset of the T/NT precue). This allowed us to identify the topo graphical distribution of the significant differences between conditions in the time windows defined on the basis of the cluster based running Wilcoxon test. The topographical EEG power differences between con ditions in each time window and frequency band were analyzed by means of nonparametrical Wilcoxon signed rank tests (one sided) using all 63 electrode sites. For PLVs, Wilcoxon signed rank tests (one sided) were calculated for each electrode pair. PLVs between 2 elec trode sites were considered significant when PLVs differed with $P<$ 0.005 between conditions. A randomization approach was used to correct for multiple comparisons across electrode sites (Blair and Kar niski 1993; Hanslmayr et al. 2009). Here, it is tested how often the result of the first step can be obtained when randomly shuffling values between conditions in 1000 iterations. The resulting probability is denoted as $P_{\text {corr }}$.

Source statistics of power were calculated using dependent $t$ tests for the comparison between conditions $(P<0.05$, one tailed) in time windows of $1 \mathrm{~s}$ ( $01,1.52 .5 \mathrm{~s}$; see above). Analyses were corrected for multiple comparisons using a cluster based permutation approach (Maris and Oostenveld 2007).

In order to reveal a possible relationship between brain activity and subsequent forgetting, we divided our participants into high and low forgetters by a median split based on NT baseline forgetting scores within the 6 counterbalancing groups. We employed independent $t$ tests for comparing NT T differences in EEG power between high and low forgetters at the source level $(P<0.05$, one tailed). Analyses were corrected for multiple comparisons using a cluster based permu tation approach (Maris and Oostenveld 2007). We also contrasted PLV at the selected center frequencies between the 2 subgroups. Differ ences between subgroups at the scalp level were always tested by means of nonparametric Mann Whitney $U$ tests that were controlled for multiple comparisons through randomization (Blair and Karniski 1993; Hanslmayr et al. 2009). Between group analyses were restricted to time windows and frequency bands that yielded significant effects in the overall analysis. Following the same approach, based on the overall results, we also investigated the relationship between phase synchron ization and power. Median splits on power and PLV values were per formed on the mean difference values between $\mathrm{T}$ and NT conditions for each subject, taking into account only those electrodes/electrode pairs that were significant in the overall analysis comparing $\mathrm{T}$ and NT conditions in the whole sample.

\section{Results}

\section{Behavioral Results}

As reported in our previous study (Hanslmayr et al. 2009), recall in the NT condition $(84.4 \%, \mathrm{SD}=19.4)$ was significantly reduced when compared with baseline $(93.4 \%, \mathrm{SD}=14.4$; $\left.t_{(23)}=3.08 ; P<0.005\right)$. Recall in the $\mathrm{T}$ condition $(93.8 \%, \mathrm{SD}=$ 9.5) did not differ significantly from baseline $\left(t_{(23)}=0.10\right.$; $P>0.5)$.

\section{EEG Oscillations}

In order to identify time windows and frequency bands of interest, we inspected EEG power differences between NT and $\mathrm{T}$ conditions by means of continuous Wilcoxon signed rank tests, averaged across all electrode sites (Fig. 1B). Seven clus ters emerged in the time frequency space of which 3 survived the permutation statistic. The biggest cluster started at $1.45 \mathrm{~s}$ and lasted until the end of the epoch, comprising significant effects in all frequency bands from 5 to $19 \mathrm{~Hz}(P<0.05)$. The second cluster ranged from 0.7 to $1.15 \mathrm{~s}$ and 8 to $19 \mathrm{~Hz}(P<$ $0.025)$. The third cluster ranged from 0.05 to $0.45 \mathrm{~s}$ and from 5 to $8 \mathrm{~Hz}(P<0.017)$. Based on these findings, 3 time windows of interest were selected to further investigate the topographic al distribution of effects, $00.5,0.51 .0$, and $1.53 .0 \mathrm{~s}$ after the T/NT Cue. We focused on the same frequency bands and time windows as in the EEG power analyses to reveal patterns of inter regional communication by comparing indices of elec trode phase coupling (PLV) between T and NT conditions.

\section{T/NT Cue Related Effects}

\section{Power}

With onset of the T/NT Cue, we observed an early ( $\left.\begin{array}{lll}0 & 0.5 & \mathrm{~s}\end{array}\right)$ in crease in theta $(59 \mathrm{~Hz})$ power for the NT condition. This early 
increase in theta power was evident over frontal and parietal electrode sites, and was driven by a significant increase of theta power in the NT condition when compared with the T condition as revealed in a nonparametric Wilcoxon test $\left(P_{\text {corr }}<\right.$ 0.05; Fig. $2 A$, left). No significant effects in alpha and beta power were observed in this time window $\left(P_{\text {corr }}>0.10\right)$. In the subsequent time window $(0.51 .0 \mathrm{~s})$, the theta power effect was reversed, with the NT condition now showing decreased power relative to the $\mathrm{T}$ condition $\left(P_{\text {corr }}<0.05\right.$; Fig. $2 A$, right). Baseline corrected source activity at $7 \mathrm{~Hz}$ was contrasted between $\mathrm{T}$ and NT conditions for the whole time interval fol lowing the T/NT Cue ( $\left.\begin{array}{lll}0 & 1.0\end{array}\right)$. This analysis revealed that the theta power increase in the NT condition was driven by sources in the left dorsolateral prefrontal cortex, correspond ing to BA 9/46 (Fig. 2B), and also comprising portions of the anterior cingulate cortex (BA 32).

Central to our hypothesis, we explored whether the reported theta power effects on the source level were functionally rele vant for forgetting. To this end, we tested whether EEG power differences between conditions (NT T) differed between high and low forgetters. A stronger prefrontal theta power increase $(\mathrm{NT}>\mathrm{T})$ was expected for high forgetters compared with low forgetters in the T/NT Cue interval, indicating efficient cognitive control. High forgetters showed a stronger theta power increase $(\mathrm{NT}>\mathrm{T})$ compared with low forgetters in the PFC, comprising the left and right middle frontal gyrus ( $\sim \mathrm{BA} 8 / 9 / 46$, Fig. $2 C)$. In the same time window, high forgetters also showed theta power decreases in the left inferior temporal gyrus ( BA 20/37), left fu siform gyrus ( $\sim$ BA 37 ), left parahippocamapal gyrus ( $\sim$ BA 36), the left inferior parietal lobe (BA 40 ), and the primary visual cortex ( BA 17/18, Fig. 2C).

Significant power effects during the T/NT Cue interval also emerged in the other 2 frequency bands. In the alpha and beta band, significantly less power in the NT compared with the T condition was observed in the time window of $0.51 .0 \mathrm{~s}$ after the T/NT Cue (both $P_{\text {corr }}<0.05$ ). The alpha effect had a largely left hemispheric distribution, whereas the beta difference dis played a more parietal effect on the scalp (Fig. 2D,E).

Source localization of these effects most prominently re vealed large decreases of alpha and beta oscillatory power in the prefrontal cortex and additional foci in the MTL (alpha) and the visual cortex (beta; Supplementary Fig. $1 A$ and Supple mentary Material, Results). Comparing high and low forgetters revealed differences in power in the dorsal parietal cortex, an terior cingulate cortex, and left MTL (Supplementary Fig. $1 B$ and Supplementary Material, Results).

\section{Phase Synchronization}

With onset of the T/NT Cue, a significant increase in phase syn chronization emerged in the alpha frequency band for the NT condition compared with the T condition $\left(P_{\text {corr }}<0.01\right.$, Fig. $3 A$, middle). Increased phase synchronization was observed between frontal and parietal/occipital electrodes, and also at parietal and occipital electrode pairs. Such an increase in
A

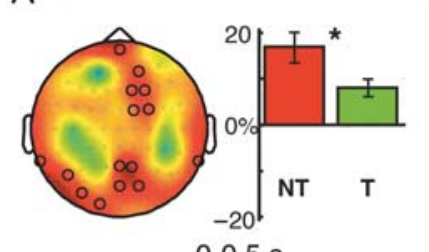

$0-0.5 \mathrm{~s}$
Theta

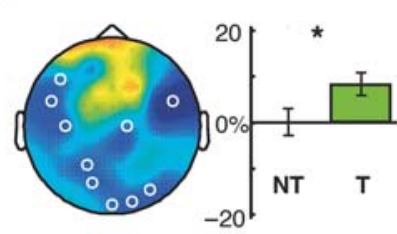

$0.5-1 \mathrm{~s}$
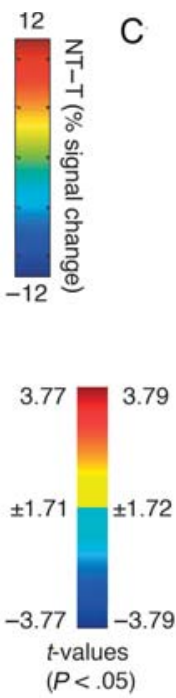

$(P<.05)$

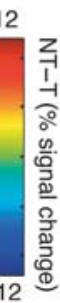

C
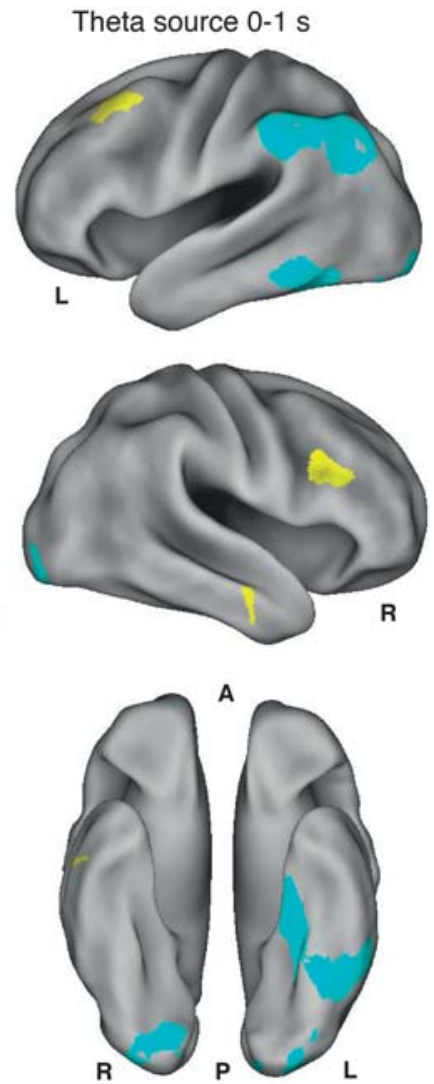

Figure 2. Significant $\left(P_{\text {corr }}<0.05\right)$ EEG power differences between NT and T conditions in the theta $(A)$, alpha $(D)$, and beta $(E)$ frequency bands for the 0 o.5 and $0.51 \mathrm{~s}$ time windows following the T/NT Cue. Headplots depict topographical distribution of NT T power differences, and bar graphs depict power values within conditions at the significant electrode sites. Black (white) circles indicate electrodes for which NT $>$ T (NT $<$ T) power in the respective time frequency windows. Significant overall effects are marked by asterisks. Significant $(P<0.5)$ NT T theta $(7 \mathrm{~Hz})$ power differences at the source level $(B)$ between conditions $(\mathrm{NT}$ T) and $(C)$ high and low forgetters $(\mathrm{NT}$ T) for the 0 1 s time window. 
A
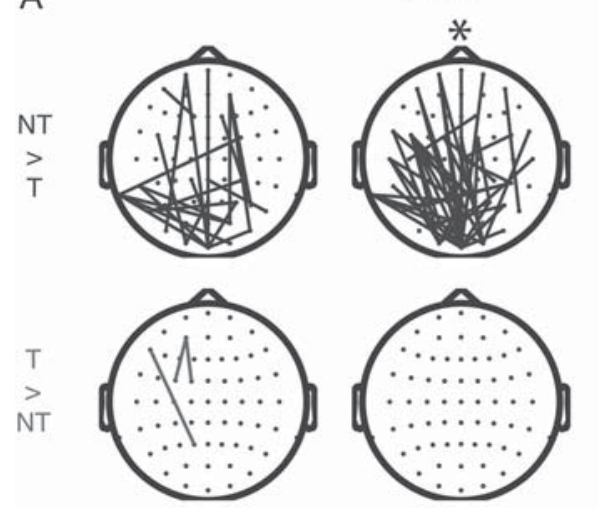

B

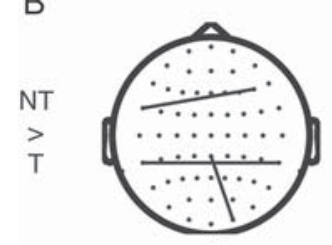

$0.5-1 \mathrm{~s}$

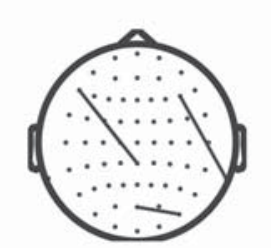

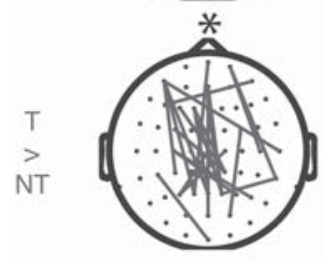

Theta

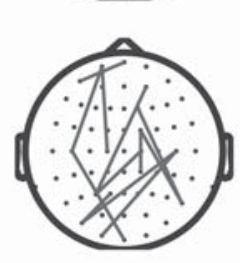

Alpha
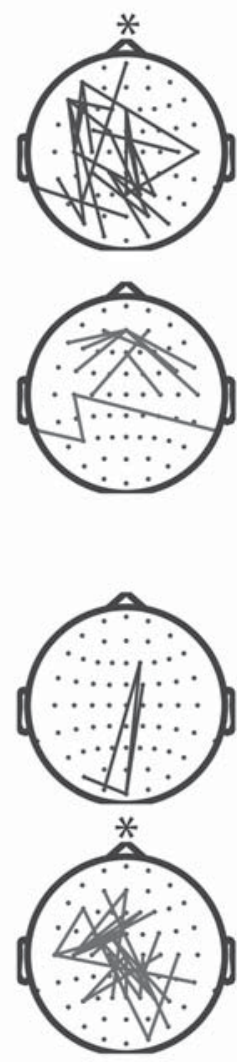

Beta
C

High $>$ low theta power

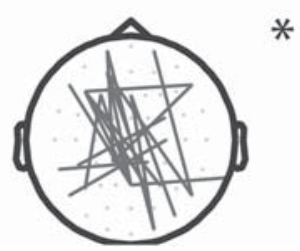

PLV $12 \mathrm{~Hz}$

$0-0.5 \mathrm{~s}$

Figure 3. PLV differences between NT and T conditions in the $00.5 \mathrm{~s}(A)$ and $0.51 \mathrm{~s}$ $(B)$ time windows. Lines display electrode pairs for which significant $(P<0.005)$ differences in PLVs were obtained between conditions (NT $>T$ upper rows, T > NT lower rows). (C) T/NT Cue related (0 $0.5 \mathrm{~s})$ alpha (12 Hz) PLV difference (NT T) between subjects showing high and low theta power differences in the same time window. Significant overall effects $\left(P_{\text {corr }}<0.05\right)$ are marked by asterisks.

phase synchronization for NT trials was also observable in the beta frequency band ( $P_{\text {corr }}<0.05$, Fig. $3 A$, right), but did not reach significance in the theta frequency band $\left(P_{\text {corr }}>0.05\right.$, Fig. $3 A$, left). In the following time window, from 0.5 to $1.0 \mathrm{~s}$, the difference between $\mathrm{T}$ and NT conditions reversed (Fig. 3B, lower row). Here, we observed a decrease in phase synchron ization for the NT condition in the theta frequency band ( $P_{\text {corr- }}$ $<0.05$ ), mostly between frontal and parietal electrodes, and in the beta frequency band $\left(P_{\text {corr }}<0.05\right)$, between frontal and central and between central and parietal electrodes.

High and low forgetters were also contrasted based on their NT T differences in phase synchronization. In the T/NT Cue interval ( $\left.\begin{array}{ll}0 & 0.5\end{array}\right)$, no significant differences in phase synchron ization in any of the 3 frequencies were observed. In addition, we tested whether alpha phase coupling and theta power in creases in the T/NT Cue interval were also related. This was

done by splitting the sample based on median theta power dif ference (NT T, $00.5 \mathrm{~s}$ ) and contrasting alpha PLV differences (NT T, $00.5 \mathrm{~s}$ ) between the 2 groups. The results show that subjects with high theta power differences also showed higher differences in the alpha PLV compared with those with low theta power differences $\left(P_{\text {corr }}<0.05\right.$; Fig. $\left.3 C\right)$, mostly between frontal and parietal electrodes.

\section{Reminder Related Effects}

\section{Power}

A pronounced decrease in theta power (no think $<$ think) was evident, starting around $0.5 \mathrm{~s}$ after presentation of the remind er (1.5 $3.0 \mathrm{~s}$ following the T/NT Cue; Fig. 1B). This theta power difference was widespread, affecting almost all electro des $\left(P_{\text {corr }}<0.001\right.$, Fig. $\left.4 A\right)$. Source localization of this theta power decrease for the NT condition $(1.52 .5 \mathrm{~s})$ revealed a dis tributed network of neural generators (Fig. $4 B$ ). In the left hemisphere, decreased theta power was evident in the fusi form gyrus, the superior temporal gyrus, and the postcentral gyrus ( BA 37/22/40/42/43). Reduced theta power in the right hemisphere was localized to the superior temporal gyrus close to the temporal pole ( $\sim \mathrm{BA} 22 / 38)$ and to superior tem poral and inferior parietal regions ( $\sim \mathrm{BA} 40)$. In addition, we identified decreased theta power in the precentral gyrus (BA 4) and the right lateral PFC.

A stronger decrease in theta power $(\mathrm{NT}<\mathrm{T})$ for high forget ters in MTL and posterior sensory processing regions was ex pected, as this should indicate successful suppression of retrieval related activity. Pronounced decreases in theta power $(\mathrm{NT}<\mathrm{T})$ in the left hemisphere were observed for high com pared with low forgetters (Fig. $4 \mathrm{C}$ ). These effects were evident in the inferior and medial temporal cortex, being most pro nounced in the parahippocampal cortex ( BA 35) and the fusi form gyrus ( BA 20, Fig. $4 C$ ).

Power decreases were not restricted to the theta frequency range. Power in the alpha $\left(P_{\text {corr }}=0.009\right)$ and beta $\left(P_{\text {corr }}>\right.$ 0.001 ) bands displayed a similar pattern, exhibiting less power in the no think compared with the think condition (Fig. $4 D, E$ ). Source localization of these effects revealed a decrease of alpha and beta oscillatory power in the prefrontal cortex and in the visual cortex for the beta frequency band (Supplemen tary Fig. $2 A$ and Supplementary Material, Results). The com parison between high and low forgetters showed alpha and beta power increases in the dorsal parietal, prefrontal, and an terior cingulate cortices (Supplementary Fig. $2 B$ and Supple mentary Material, Results). In addition, we observed increased alpha and beta oscillatory power in retrieval processing regions for high forgetters such as the inferior parietal cortex, occipital cortex, and the MTL.

\section{Phase Synchronization}

In the reminder related time window, a 2 fold phase synchron ization pattern emerged. A pronounced PLV decrease for the NT condition arose in all 3 frequency bands (all $P_{\text {corr }}<0.001$, Fig. $5 A$, lower row). Decreased phase synchronization was widespread, comprising reduced interhemispheric and fronto parietal long range coupling and also reduced short distance coupling between electrodes. At the same time, we also ob served an increase in phase synchronization for the no think condition in the alpha and beta frequency band (both $P_{\text {corr }}<$ 0.05 , Fig. $5 A$, upper row) that was most pronounced between 
A

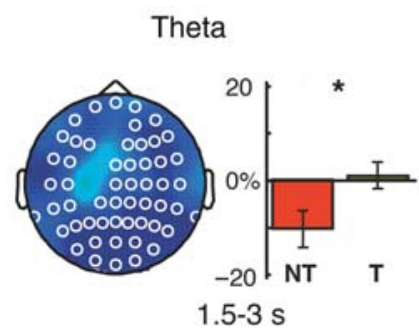

B

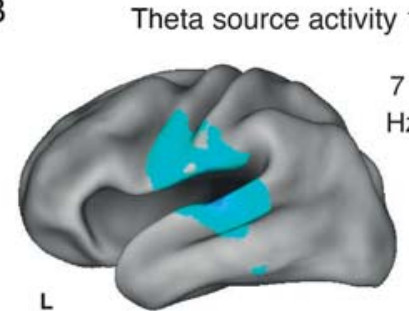

Theta source activity $1.5-2.5 \mathrm{~s}$

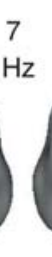

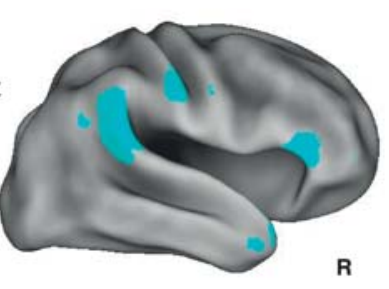

R

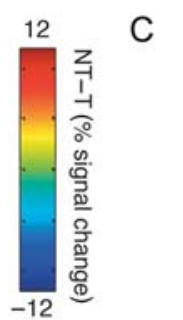

C
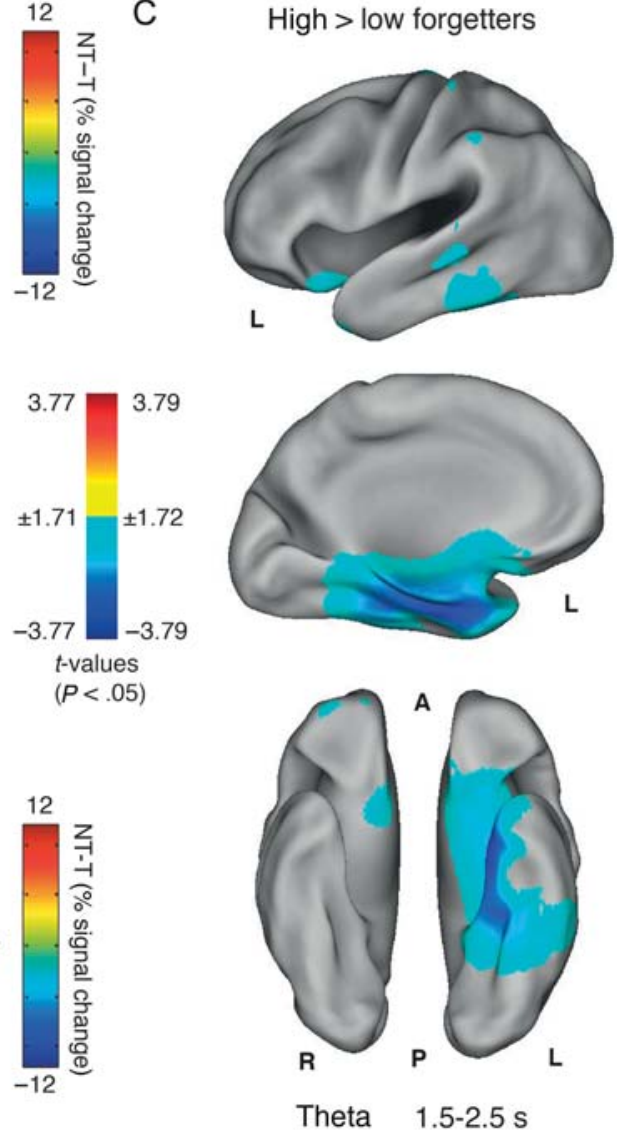
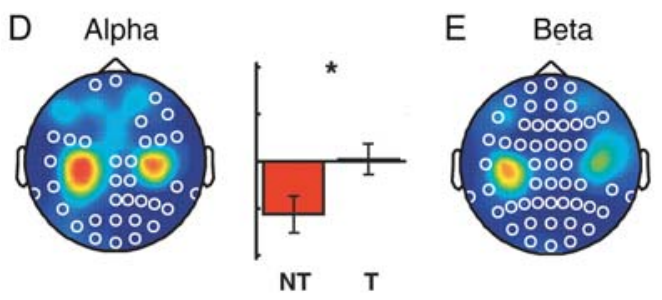

$1.5-3 \mathrm{~s}$

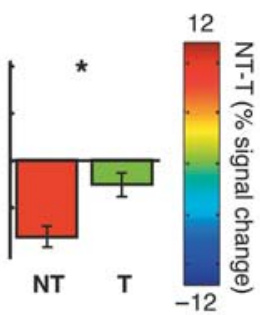

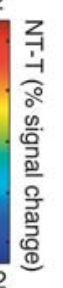

Figure 4. Significant $\left(P_{\text {corr }}<0.05\right)$ EEG power differences between NT and T conditions in the theta $(A)$, alpha $(D)$, and beta $(E)$ frequency bands following the reminder stimulus from 1.5 to $3 \mathrm{~s}$. Headplots depict topographical distribution of NT T power differences and bar graphs depict power values within conditions at the significant electrode sites. White circles indicate electrodes for which power in the NT condition is lower than for the T condition. Significant overall effects are marked by asterisks. Significant $(P<0.5)$ NT T theta $(7 \mathrm{~Hz})$ power differences at the source level $(B)$ between conditions (NT T) and $(C)$ high and low forgetters (NT T) for the $1.52 .5 \mathrm{~s}$ time window.

frontal/frontocentral and centroparietal electrodes. No NT related phase synchronization increase was observed in the theta band $\left(P_{\text {corr }}>0.5\right)$.

During the reminder interval (1.5 $3 \mathrm{~s}$ ), phase synchroniza tion at $7 \mathrm{~Hz}$ was lower in the NT compared with the T condi tion for high forgetters relative to low forgetters $\left(P_{\text {corr }}<0.01\right.$; Fig. $5 B$ ). No differences at the alpha and beta center frequen cies emerged in this comparison.

Due to the presumed role of alpha phase synchronization in top down inhibitory control (Klimesch 2012), we expected alpha phase synchronization to be involved in the down regulation of memory related activity. Therefore, we tested whether higher alpha PLVs predict lower theta power in the reminder related interval as a marker of reduced retrieval processing. We per formed a median split based on mean alpha PLV differences (NT T) and compared subjects with high PLV with those exhibit ing low PLV differences. Confirming our hypothesis, subjects with high NT $\mathrm{T}$ alpha phase coupling showed stronger theta power differences (NT T) at $7 \mathrm{~Hz}$ in the same time window $\left(P_{\text {corr- }}\right.$ $<0.001$; Fig. $5 C$ ). This suggests that alpha phase coupling is related to a decrease of reminder related theta power.

\section{Discussion}

The current study sheds light on the brain oscillatory dynamics underlying voluntary memory suppression in the T/NT task (Anderson and Green 2001). Using a modified version of the
T/NT paradigm (Hanslmayr et al. 2009; Hanslmayr, Leipold, et al. 2010), we were able to temporally segregate the oscilla tory correlates of the initiation of cognitive control processes as triggered by the T/NT cue, from the actual effects of the sup pression of specific target memories. Consistent with our hy pothesis, 2 main findings emerged: first, upon presentation of the T/NT cue, higher prefrontal theta power was triggered by the NT compared with the T condition. This pattern was paral leled in fronto parietal alpha phase synchronization. Secondly, upon presentation of the reminder stimulus, a sustained and widespread decrease in theta power and phase synchroniza tion was evident, localized to posterior sensory regions and the MTL when comparing high with low forgetting subjects. These results confirm and extend previous EEG (Depue et al. 2013; Ketz et al. 2014) and fMRI studies (Anderson et al. 2004; Depue et al. 2007; Benoit and Anderson 2012) in suggesting a dynamic interaction between a prefrontal cognitive control network and a hippocampo cortical memory storage network (Anderson and Hanslmayr 2014). In the following, we will discuss how activity in each of these networks and in their interaction is reflected in the reported results. We will also discuss the effects in the other frequency bands that were not expected (e.g., the beta frequency band).

\section{Control Network}

We observed a pronounced increase in theta power over frontal and parietal electrode sites immediately after delivery 
A

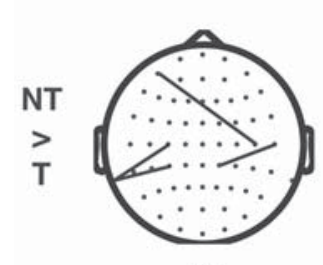

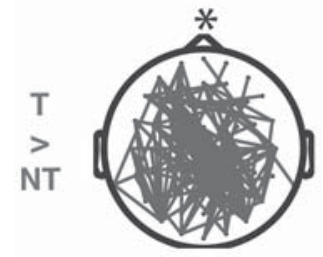

Theta
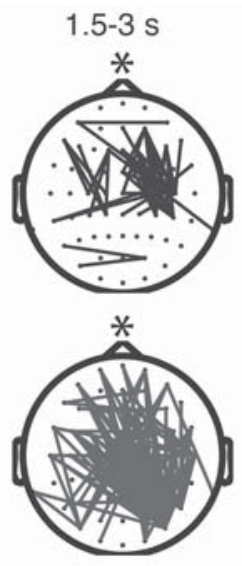

Alpha
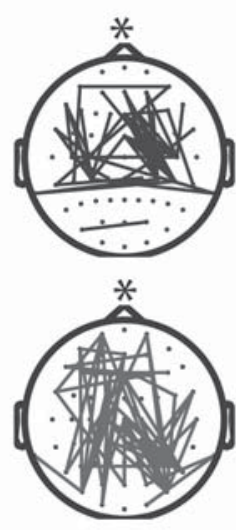

Beta
B

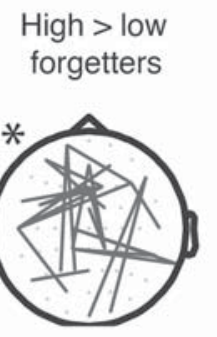

PLV $7 \mathrm{~Hz}$

1.5-3 s
C High < low alpha PLV

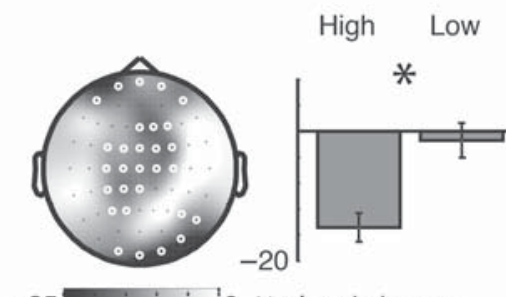

$-25 \div \quad 0 \%$ signal change

Power $7 \mathrm{~Hz} 1.5-3 \mathrm{~s}$
Figure 5. (A) PLV differences between NT and T conditions in the $1.53 \mathrm{~s}$ time window. Lines display electrode pairs for which significant $(P<0.005)$ differences in PLVs were obtained between conditions (NT $>\mathrm{T}$ upper row, $\mathrm{T}>\mathrm{NT}$ lower row). (B) Reminder related $(1.53 \mathrm{~s})$ theta $(7 \mathrm{~Hz}) \mathrm{PLV}$ differences between high and low forgetters. Significant overall effects $\left(P_{\text {corr }}<0.05\right)$ are marked by asterisks. (C) Reminder related $(1.53 \mathrm{~s})$ theta $(7 \mathrm{~Hz})$ power differences between subjects showing high and low alpha PLV differences in the same time window.

of the NT cue. Increases in theta power with a similar frontal and parietal distribution on the scalp have previously been re ported in T/NT studies (Depue et al. 2013; Ketz et al. 2014) and other forgetting paradigms (Hanslmayr, Staudigl, et al. 2010; Staudigl et al. 2010; Waldhauser, Johansson, et al. 2012). More generally, similar prefrontal theta power effects were observed in cognitive control tasks that require interference processing and conflict adaptation (Hanslmayr et al. 2008; Pas tötter et al. 2013). In line with these studies, the reported theta power increases in response to the NT cue were localized to the medial and lateral prefrontal cortex. fMRI studies employ ing the T/NT paradigm report activity in similar brain regions, although these studies highlight the role of the right lateral PFC in contrast to our finding of mostly left lateralized and medial sources (Anderson et al. 2004; Depue et al. 2007; Benoit and Anderson 2012). It is possible that EEG beamform ing, in contrast to fMRI, failed to identify right PFC sources in the overall analysis, since it can have problems to detect bilat erally correlated sources (Dalal et al. 2006). However, contrast ing high versus low forgetters in our study revealed a clear activation in the right lateral PFC. This suggests that the left PFC, together with structures such as the anterior cingulate cortex, serves to generally initiate executive control, whereas the right $\mathrm{PFC}$ is the decisive brain region in the success ful direct suppression of unwanted memories (Benoit and
Anderson 2012). Strikingly, the theta effect occurred before presentation of the actual reminder stimulus. Thus, theta effects appear to be independent of the reactivation of the to be suppressed memory representation (cf. Anderson et al. 2004; Hanslmayr, Staudigl, et al. 2010; Staudigl et al. 2010; Waldhauser, Johansson, et al. 2012). In line with our previous ERP study, our data suggest that the observed cognitive control mechanisms can be triggered voluntarily (Hanslmayr et al. 2009). This active control process appears to reflect a prepar ation for upcoming episodic inhibition, leading to later forget ting of the target items. Such a result is in line with studies on task switching, showing that the execution of an upcoming task can be initiated by the presentation of a preceding indica tive cue (e.g., Nicholson et al. 2005).

Increases in prefrontal theta power were paralleled by in creases in long range phase synchronization in the alpha and beta frequency band. Intriguingly, subjects who exhibited high theta power in response to the T/NT cue also showed high alpha phase synchronization in the same time window. The two effects therefore appear to be linked, and both likely reflect the action of a top down control network, which pre pares the brain to suppress the memory associated with an up coming reminder. Enhanced alpha phase synchronization was not restricted to the T/NT cue interval, however. Alpha phase synchronization remained at a high level also during the pres entation of the reminder, correlating on a between subject level with decreased theta power in the same time window. This latter result is suggestive of a sustained top down fronto parietal control network (Sadaghiani et al. 2012; Paz Alonso et al. 2013; Sauseng et al. 2013) in the service of suppressing theta synchrony in memory related networks, which would otherwise readily reinstate the unwanted memory trace (Fuen temilla et al. 2014). Importantly, the timing of the late effect roughly coincides with ERP effects related to cognitive control that were reported in previous studies to immediately precede and sustain a reduction of recollection (Bergström et al. 2009; Mecklinger et al. 2009; Waldhauser, Lindgren, et al. 2012).

\section{Memory Network}

Suppression of specific memories was associated with a pronounced decrease in theta power, starting $\sim 0.5 \mathrm{~s}$ after pres entation of the reminder. Importantly, successful memory re trieval is typically associated with increased oscillatory power in the theta frequency band (Nyhus and Curran 2010). En hanced theta power during memory tasks was source localized to the MTL in various MEG studies (Guderian et al. 2009; Stau digl and Hanslmayr 2013). Comparing high and low forgetters on the source level showed that high forgetting subjects were indeed characterized by a pronounced decrease of theta power in MTL regions (Fig. $4 C$ ). Although source localization results, especially from EEG data, should always be interpreted with caution, these results converge with previous fMRI studies (An derson et al. 2004; Depue et al. 2007; Benoit and Anderson 2012). Voluntary suppression of memories therefore seems to induce a decrease of theta power in retrieval related brain net works, which correlates with later forgetting. Although the contrast between NT and T conditions across all subjects did not reveal reduced theta power in the MTL, the regions ob served in the overall contrast overlap with areas revealed in previous fMRI studies which also report decreased BOLD in the superior and medial temporal gyri, the parietal cortex, and 
the fusiform gyrus (Anderson et al. 2004; Depue et al. 2007; Benoit and Anderson 2012).

Pronounced reductions were also evident in theta phase synchronization, suggesting that memory suppression affects a larger network of brain areas involved in the retrieval and post retrieval processing of the target memory. This finding sug gests that not only local synchronization (power) is reduced, but also communication between brain areas involved in memory processing. In line with the reduced power in the MTL, this result could reflect a reduction of synchrony within hippocampo cortical feedback loops (Guderian and Düzel 2005; Cashdollar et al. 2009).

In addition to the suppression effects in the reminder inter val, we observed a decrease in theta power and phase syn chronization already preceding the onset of the reminder in the NT condition when compared with the T condition. This is in line with our previous study (Hanslmayr et al. 2009), sug gesting a preparatory effect triggered by the T/NT cue. The dif ference between $\mathrm{T}$ and NT conditions appears to be driven by a relative increase in theta power for the $\mathrm{T}$ condition, as opposed to below baseline power for the NT condition. In creased theta power before the onset of a reminder stimulus has been shown to reflect enhanced memory related process ing (Addante et al. 2011). Our data suggest that subjects prepare for retrieval following a think cue, while they success fully avoid retrieval preparation in the no think condition (Hanslmayr et al. 2009). Although such an interpretation seem ingly speaks against active inhibitory effects, the anticipatory avoidance of retrieval processing was related to later forget ting. High forgetters displayed reduced theta power in inferior temporal, ventral parietal, and occipital areas before onset of the face stimulus, suggesting that the early decrease in theta power is a correlate of reduced visual and bottom up attentional processing of the reminder stimulus in the NT con dition relative to the $\mathrm{T}$ condition for high forgetters when com pared with low forgetters (Depue et al. 2007; Cabeza 2008). Such a mechanism could be the reason for why memory sup pression can be boosted by giving an anticipatory cue, before the reminder appears (Hanslmayr, Leipold, et al. 2010).

\section{Effects in Other Frequency Bands}

Apart from the reported results confirming our hypotheses, additional time frequency patterns arose. The power decrease during and before the reminder interval was not restricted to the theta frequency band, but was also observed in the alpha and beta frequency range. As evident from the source localiza tion results, the power decrease in the alpha and beta fre quency band was generated by cortical sources quite different from the theta effects. Alpha and beta power decreased in frontal, temporal, and occipital regions in the NT task when compared with the $\mathrm{T}$ condition. Recent studies point to an inverse relationship between alpha/beta power and BOLD response (Hanslmayr et al. 2011; Scheeringa et al. 2011). In this line, the occipital decrease in beta power could reflect the higher visual processing of the NT cue and the reminder stimulus, as a response to the instruction in the experiment to focus on the cue and stimulus instead of retrieving the target memory. The prefrontal decrease of alpha and beta power could be a correlate of higher activation in top down control areas, including the inferior frontal gyrus, a region that is in volved in inhibitory control (Aron et al. 2004; Swann et al.
2009). The anticipatory power decrease in the left temporal lobe during the T/NT cue interval could reflect preparation of MTL regions for the upcoming suppression task, or retrieval of instructions associated with the cue, or even the retrieval of substitutional memories, but this effect is difficult to be inter preted at present.

The comparison between high and low forgetters revealed increased alpha and beta power in the anterior cingulate cortex and the dorsal parietal cortex, regions involved in error monitoring and top down control of memory retrieval (Menon et al. 2001; Cabeza et al. 2008), with the latter region known to operate in the alpha frequency band (Capotosto et al. 2009). These sources converge with fMRI studies investigating the control of memory intrusions in the T/NT paradigm (Levy and Anderson 2012). Remarkably, we also observed an increase in alpha and beta power in MTL and inferior parietal areas for high forgetters during both T/NT cue and reminder intervals. Taken together with the findings on phase synchronization, these effects suggest the active inhibition of retrieval related brain areas via modulations of the alpha and beta frequency bands (Waldhauser, Johansson, et al. 2012).

Taken together, the findings on alpha and beta power differ ences between conditions and high and low forgetters point to additional processes that might mediate intentional memory suppression. This being said, it has to be noted that the ob served effects were less clear and more scattered than the theta power effects. The interplay of increased and decreased alpha and beta power in the various regions and its role for suppression induced forgetting deserves further investigation in future studies.

A similar ambiguous pattern was observed in phase syn chronization measures. PLVs were not only decreased in the theta frequency range, but were also decreased in the alpha and beta range. Phase synchronization in the alpha frequency band is assumed to reflect neural timing that can enhance memory related processing and facilitate communication between brain areas (Klimesch et al. 2008). Interestingly, alpha and beta phase de synchronization has recently been found during intentional forgetting paradigms (Bäuml et al. 2008; Hanslmayr et al. 2012; Sauseng et al. 2013), which has been in terpreted as an effective mechanism to decrease synaptic plas ticity in the service of suppressing irrelevant memories (Anderson and Hanslmayr 2014). In line with theoretical notions suggesting that alpha oscillations play a complex role in gating neural timing that can have both detrimental as well as enhancing effects on communication between cell assem blies (Klimesch et al. 2007), our data suggest a two fold role of alpha phase synchronization in orchestrating memory suppres sion. Future studies will have to clarify how increases of alpha/ beta synchronization as correlates of enhanced top down control can be separated from decreased synchronization in the same frequency band as a marker of reduced memory processing.

\section{Conclusions}

Replicating and extending previous EEG studies and conver ging with fMRI research, our study shows that voluntary memory suppression is the result of the dynamic interaction between a cognitive control network and a hippocampo cortical memory storage network. The suppression of unwant ed memories is achieved by the rapid recruitment of cognitive 
control, reflected in high prefrontal theta power. Sustained cognitive control is mediated by a network operating in the alpha/beta frequency range, arguably involved in monitoring and coordinating the avoidance of memory retrieval during the presentation of the reminder. The effect of this suppression of specific memories is evident in decreased local and wide spread theta synchrony, likely reflecting the active down regulation of a hippocampo cortical memory storage network.

\section{Supplementary Material}

Supplementary material can be found at: http://www.cercor. oxfordjournals.org/.

\section{Funding}

This work was supported by grants from the Swedish Research Council (VR; 4352011 7163) awarded to G.T.W. and the German Research Council (DFG; HA 5622/1 1) awarded to S.H.

\section{Notes}

Conflict of Interest: None declared.

\section{References}

Addante RJ, Watrous AJ, Yonelinas AP, Ekstrom AD, Ranganath C. 2011. Prestimulus theta activity predicts correct source memory retrieval. Proc Natl Acad Sci USA. 108:10702 10707.

Anderson MC, Green C. 2001. Suppressing unwanted memories by executive control. Nature. 410:366 369

Anderson MC, Hanslmayr S. 2014. Neural mechanisms of motivated forgetting. Trends Cogn Sci. 18:279 292.

Anderson MC, Huddleston E. 2012. Towards a cognitive and neurobio logical model of motivated forgetting. In: Belli RF, editor. True and false recovered memories. New York: Springer. p. 53120.

Anderson MC, Ochsner KN, Kuhl B, Cooper J, Robertson E, Gabrieli SW, Glover GH, Gabrieli JD. 2004. Neural systems underlying the suppression of unwanted memories. Science. 303:232 235.

Aron AR. 2007. The neural basis of inhibition in cognitive control. Neuroscientist. 13:214 228.

Aron AR, Robbins TW, Poldrack RA. 2004. Inhibition and the right in ferior frontal cortex. Trends Cogn Sci. 8:170 177.

Battig WF, Montague WE. 1969. Category norms of verbal items in 56 categories a replication and extension of the connecticut category norms. J Exp Psychol. 80:1.

Bäuml K HT, Hanslmayr S, Pastötter B, Klimesch W. 2008. Oscillatory correlates of intentional updating in episodic memory. Neuro image. 41:596 604 .

Benoit RG, Anderson MC. 2012. Opposing mechanisms support the voluntary forgetting of unwanted memories. Neuron. 76:450 460 .

Bergström ZM, de Fockert JW, Richardson Klavehn A. 2009. ERP and behavioural evidence for direct suppression of unwanted memor ies. Neuroimage. 48:726 737 .

Blair RC, Karniski W. 1993. An alternative method for significance testing of waveform difference potentials. Psychophysiology. 30: 518524 .

Bulevich JB, Roediger HL, Balota DA, Butler AC. 2006. Failures to find suppression of episodic memories in the think/no think paradigm. Mem Cognit. 34:1569 1577.

Cabeza R. 2008. Role of parietal regions in episodic memory retrieval: the dual attentional processes hypothesis. Neuropsychologia. 46: 18131827.

Cabeza R, Ciaramelli E, Olson IR, Moscovitch M. 2008. The parietal cortex and episodic memory: an attentional account. Nat Rev Neurosci. 9:613 625.
Capotosto P, Babiloni C, Romani GL, Corbetta M. 2009. Frontoparietal cortex controls spatial attention through modulation of anticipatory alpha rhythms. J Neurosci. 29:5863 5872.

Cashdollar N, Malecki U, Rugg Gunn FJ, Duncan JS, Lavie N, Duzel E. 2009. Hippocampus dependent and independent theta networks of active maintenance. Proc Natl Acad Sci USA. 106:20493 8.

Corbetta M, Shulman GL. 2002. Control of goal directed and stimulus driven attention in the brain. Nat Rev Neurosci. 3:201 215.

Dalal SS, Sekihara K, Nagarajan SS. 2006. Modified beamformers for coherent source region suppression. IEEE Trans Biomed Eng. 53:1357 1363.

Depue BE, Banich MT, Curran T. 2006. Suppression of emotional and nonemotional content in memory effects of repetition on cogni tive control. Psychol Sci. 17:441 447.

Depue BE, Curran T, Banich MT. 2007. Prefrontal regions orchestrate suppression of emotional memories via a two phase process. Science. 317:215 219.

Depue BE, Ketz N, Mollison MV, Nyhus E, Banich MT, Curran T. 2013. ERPs and neural oscillations during volitional suppression of memory retrieval. J Cogn Neurosci. 25:1624 1633.

Düzel E, Habib R, Schott B, Schoenfeld A, Lobaugh N, McIntosh A, Scholz M, Heinze H. 2003. A multivariate, spatiotemporal analysis of electromagnetic time frequency data of recognition memory. Neuroimage. 18:185 197.

Fries P. 2005. A mechanism for cognitive dynamics: neuronal commu nication through neuronal coherence. Trends Cogn Sci. 9:474 480 .

Fuentemilla L, Barnes G, Düzel E, Levine B. 2014. Theta oscillations orchestrate medial temporal lobe and neocortex in remembering autobiographical memories. Neuroimage. 85:730 737.

Gross J, Kujala J, Hamalainen M, Timmermann L, Schnitzler A, Salme lin R. 2001. Tomographic mapping of functional connectivities from MEG recordings. Neuroimage. 13:S136.

Guderian S, Düzel E. 2005. Induced theta oscillations mediate large scale synchrony with mediotemporal areas during recollection in humans. Hippocampus. 15:901 912.

Guderian S, Schott BH, Richardson Klavehn A, Duzel E. 2009. Medial temporal theta state before an event predicts episodic encoding success in humans. Proc Natl Acad Sci USA. 106:5365 5370.

Hanslmayr S, Leipold P, Bäuml K HT. 2010. Anticipation boosts forget ting of voluntarily suppressed memories. Memory. 18:252 257.

Hanslmayr S, Leipold P, Pastötter B, Bäuml K HT. 2009. Anticipatory signatures of voluntary memory suppression. J Neurosci. 29: 27422747.

Hanslmayr S, Pastötter B, Bäuml K HT, Gruber S, Wimber M, Klimesch W. 2008. The electrophysiological dynamics of interference during the stroop task. J Cogn Neurosci. 20:215 225.

Hanslmayr S, Staudigl T, Aslan A, Bäuml K HT. 2010. Theta oscillations predict the detrimental effects of memory retrieval. Cogn Affect Behav Neurosci. 10:329 338.

Hanslmayr S, Volberg G, Wimber M, Oehler N, Staudigl T, Hartmann T, Raabe M, Greenlee MW, Bäuml KHT. 2012. Prefrontally driven down regulation of neural synchrony mediates goal directed forget ting. J Neurosci. 32:14742 14751.

Hanslmayr S, Volberg G, Wimber M, Raabe M, Greenlee MW, Bäuml K HT. 2011. The relationship between brain oscillations and BOLD signal during memory formation: a combined EEG fMRI study. J Neurosci. 31:15674 15680.

Ille N, Berg P, Scherg M. 2002. Artifact correction of the ongoing EEG using spatial filters based on artifact and brain signal topographies. J Clin Neurophysiol. 19:113 124.

Kayser J, Tenke CE. 2006. Principal components analysis of Laplacian waveforms as a generic method for identifying ERP generator pat terns: I. Evaluation with auditory oddball tasks. Clin Neurophysiol. 117:348 368.

Ketz N, O'Reilly RC, Curran T. 2014. Classification aided analysis of oscillatory signatures in controlled retrieval. Neuroimage. 85: 749760 .

Klimesch W. 2012. Alpha band oscillations, attention, and controlled access to stored information. Trends Cogn Sci. 16:606 617.

Klimesch W, Freunberger R, Sauseng P, Gruber W. 2008. A short review of slow phase synchronization and memory: evidence for 
control processes in different memory systems? Brain Res. 1235: 3144.

Klimesch W, Sauseng P, Hanslmayr S. 2007. EEG alpha oscillations: the inhibition timing hypothesis. Brain Res Brain Res Rev. 53:63 88.

Lachaux J P, Rodriguez E, Martinerie J, Varela FJ. 1999. Measuring phase synchrony in brain signals. Hum Brain Mapp. 8:194 208.

Levy BJ, Anderson MC. 2012. Purging of memories from con scious awareness tracked in the human brain. J Neurosci. 32: 1678516794 .

Mannhaupt H R. 1983. German category norms for verbal items in 40 categories. Mem Cognit. 2:264 278.

Maris E, Oostenveld R. 2007. Nonparametric statistical testing of EEG and MEG data. J Neurosci Methods. 164:177 190

Martinez A, Benavente R. 1998. The AR face database. Computer Vision Center, Technical Report 3.

Mecklinger A, Parra M, Waldhauser GT. 2009. ERP correlates of inten tional forgetting. Brain Res. 1255:132 147.

Menon V, Adleman NE, White CD, Glover GH, Reiss AL. 2001. Error related brain activation during a go/nogo response inhibition task. Hum Brain Mapp. 12:131 143.

Nicholson R, Karayanidis F, Poboka D, Heathcote A, Michie PT. 2005. Electrophysiological correlates of anticipatory task switching pro cesses. Psychophysiology. 42:540 554.

Nyhus E, Curran T. 2010. Functional role of gamma and theta oscilla tions in episodic memory. Neurosci Biobehav Rev. 34:1023 1035.

Oostenveld R, Fries P, Maris E, Schoffelen J M. 2011. Fieldtrip: open source software for advanced analysis of MEG, EEG, and invasive electrophysiological data. Comput Intell Neurosci. 2011:1.

Osipova D, Takashima A, Oostenveld R, Fernandez G, Maris E, Jensen O. 2006. Theta and gamma oscillations predict encoding and re trieval of declarative memory. J Neurosci. 26:7523 7531.

Pastötter B, Dreisbach G, Bäuml K HT. 2013. Dynamic adjustments of cognitive control: oscillatory correlates of the conflict adaptation effect. J Cogn Neurosci. 25:2167 2178.

Paz Alonso P, Bunge S, Anderson M, Ghetti S. 2013. Strength of coupling within a mnemonic control network differentiates those who can and cannot suppress memory retrieval. J Neurosci. 33: 50175026.
Pfurtscheller G, Aranibar A. 1977. Event related cortical desynchron ization detected by power measurements of scalp EEG. Electroen cephalogr Clin Neurophysiol. 42:817 826.

Quraan M, Moses S, Hung Y, Mills T, Taylor M. 2011. Detection and lo calization of hippocampal activity using beamformers with MEG: a detailed investigation using simulations and empirical data. Hum Brain Mapp. 32:812 827.

Sadaghiani S, Scheeringa R, Lehongre K, Morillon B, Giraud A L, D'E sposito M, Kleinschmidt A. 2012. A band phase synchrony is related to activity in the fronto parietal adaptive control network. J Neurosci. 32:14305 14310 .

Sauseng P, Gerloff C, Hummel F. 2013. Two brakes are better than one: the neural bases of inhibitory control of motor memory traces. Neuroimage. 65:52 58.

Sauseng P, Klimesch W, Doppelmayr M, Pecherstorfer T, Freunberger R, Hanslmayr S. 2005. EEG alpha synchronization and functional coupling during top down processing in a working memory task. Hum Brain Mapp. 26:148 155.

Scheeringa R, Fries P, Petersson KM, Oostenveld R, Grothe I, Norris DG, Hagoort P, Bastiaansen MC. 2011. Neuronal dynamics under lying high and low frequency EEG oscillations contribute inde pendently to the human bold signal. Neuron. 69:572 583 .

Staudigl T, Hanslmayr S. 2013. Theta oscillations at encoding mediate the context dependent nature of human episodic memory. Curr Biol. 23:1101 1106.

Staudigl T, Hanslmayr S, Bäuml K HT. 2010. Theta oscillations reflect the dynamics of interference in episodic memory retrieval. J Neu rosci. 30:11356 11362.

Swann N, Tandon N, Canolty R, Ellmore T, McEvoy L, Dreyer S, DiSano M, Aron A. 2009. Intracranial EEG reveals a time and frequency specific role for the right inferior frontal gyrus and primary motor cortex in stopping initiated responses. J Neurosci. 29:12675 12685.

Waldhauser GT, Johansson M, Hanslmayr S. 2012. Alpha/beta oscilla tions indicate inhibition of interfering visual memories. J Neurosci. 32:1953 1961.

Waldhauser GT, Lindgren M, Johansson M. 2012. Intentional suppres sion can lead to a reduction of memory strength: behavioral and electrophysiological findings. Front Psychol. 3:401. 